\title{
Application of Mathieu Functions to Solve Wave Field Changes by Elliptic Bathymetric Forms with Gradual Depth Transitions
}

\author{
Christopher J. Bender* and Robert G. Dean
}

Taylor Engineering, Inc. 10151 Deerwood Park Blvd, Bldg 300, Suite 300 Jacksonville, FL 32259, USA

University of Florida, Civil and Coastal Engineering Department, 575 Weil Hall Gainesville, FL 32611, USA

\begin{abstract}
This paper presents and demonstrates a method to determine wave field modifications resulting from elliptic bathymetric anomalies (pit or shoal) with gradual transitions in depth. The analytic (semi-numerical) method is valid for linear waves in a uniform depth domain with an arbitrary number of concentric elliptic forms of different, but uniform, depths combined to represent either a pit or a shoal. Sections present the theory, formulation, and results in the form of contour plots of the relative amplitude in the presence of the depth anomaly. The elliptic forms in the model induce wave transformation through processes of wave refraction, wave diffraction, and wave reflection with asymmetry in the solution for oblique incident wave angles. The results investigate the effect of the incident wave angle on the resulting wave field.
\end{abstract}

Keywords: Wave transformation, elliptic forms, bathymetric anomaly.

\section{INTRODUCTION}

Construction of beach nourishment projects requires large volumes of sediment often obtained by the removal from borrow pits located in reasonably shallow water. These modifications of the nearshore bathymetry have the potential to affect wave transformation processes and thus to alter the equilibrium shoreline planforms on the landward side of the borrow areas. Recent cases of erosional hot spots (EHS) associated with beach nourishment projects have increased interest in the prediction of the mechanisms of borrow pit alteration of the local wave field. Currently, a need exists for a better understanding of how borrow pit characteristics such as size and depth interact with the incident wave field to determine the effect of the pits on the nourished beach and to anticipate the effects of various designs.

An analytical (semi-numerical) solution to the threedimensional pit/shoal problem is obtained using the general method of Takano [1] for an elliptical pit/shoal. The method develops gradual transitions in depth as a series of steps of uniform depth as in Bender and Dean [2,3] where axisymmetric forms with gradual transitions were studied. The present method employs a series summation of Mathieu functions for the case of an elliptic depth anomaly instead of the series summation of Bessel functions as in the axisymmetric case of Bender and Dean [3].

The analytic model developed within this study will not replace or supersede the more complex numerical schemes that allow arbitrary bathymetry and more complex waverelated processes, but provides an alternative model with an analytic (semi-numerical) solution.

*Address correspondence to this author at the Taylor Engineering, Inc. 10151 Deerwood Park Blvd, Bldg 300, Suite 300 Jacksonville, FL 32259, USA; Tel: 904-731-7040; Fax: 904-731-9847;

E-mail: cbender@taylorengineering.com

\section{PREVIOUS STUDIES}

Combe and Soileau [4] and Williams [5] — at full scale - and Horikawa et al. [6], Motyka and Willis [7], and Williams [5] —in the laboratory — have shown the significance of borrow pit effects on adjacent shoreline planforms. The capability to predict the effects of borrow pits on the landward shoreline has not been demonstrated.

A number of investigators employing various methods have studied the problem of waves propagating over a finite pit of uniform depth - Black and Mei [8], Williams [9], Williams and Vasquez [10], McDougal et al. [11]. These studies have shown that a significant wave shadow zone can occur landward of the pit with a standing wave pattern seaward of the pit. Bender and Dean [3] have extended previous work by developing a semi-numerical method that allows for axisymmetric bathymetric anomalies with gradual transition in depth as a series of steps of uniform depth. Incorporating an analytic one-line shoreline change model has demonstrated possible equilibrium shoreline planforms in the lee of such depth anomalies. The capability to model elliptic forms with gradual depth transitions allows investigation of depth anomalies of more natural shape (i.e. other than axisymmetric and/or vertical sidewalls).

Little previous work in the coastal engineering literature employs Mathieu functions or elliptic coordinates. Chen and Mei [12] investigate exciting forces and moments of incident waves on vertical cylinders with a finite draft and elliptical cross section. The article develops solutions via numerical application of Mathieu functions and includes the formulation of the diffraction problem for long waves in elliptic coordinates.

A recent Gutiérrez-Vega et al. [13] study provides a visual analysis of general Mathieu function behavior with several applications including water waves traveling in elliptic 


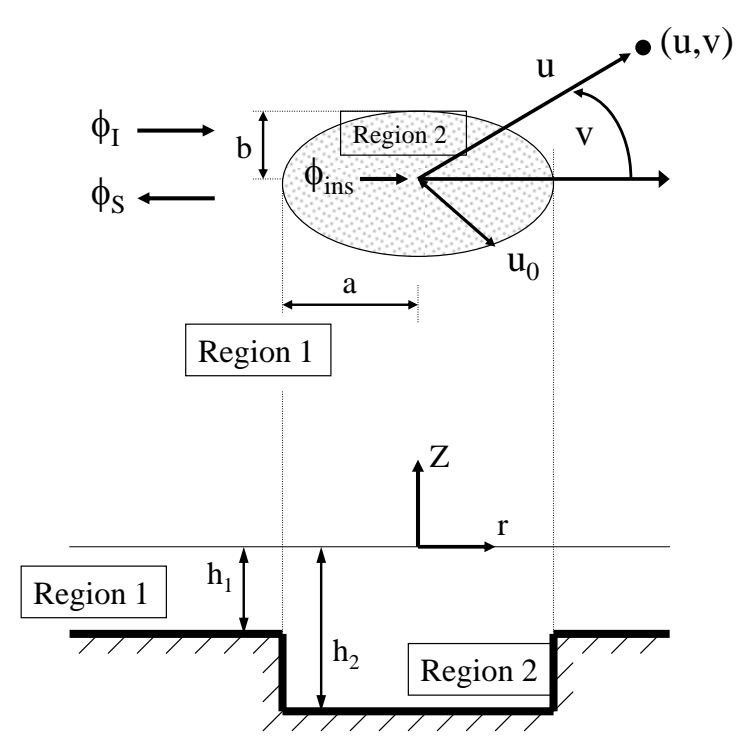

Fig. (1). Definition sketch for elliptic pit with abrupt depth transitions.

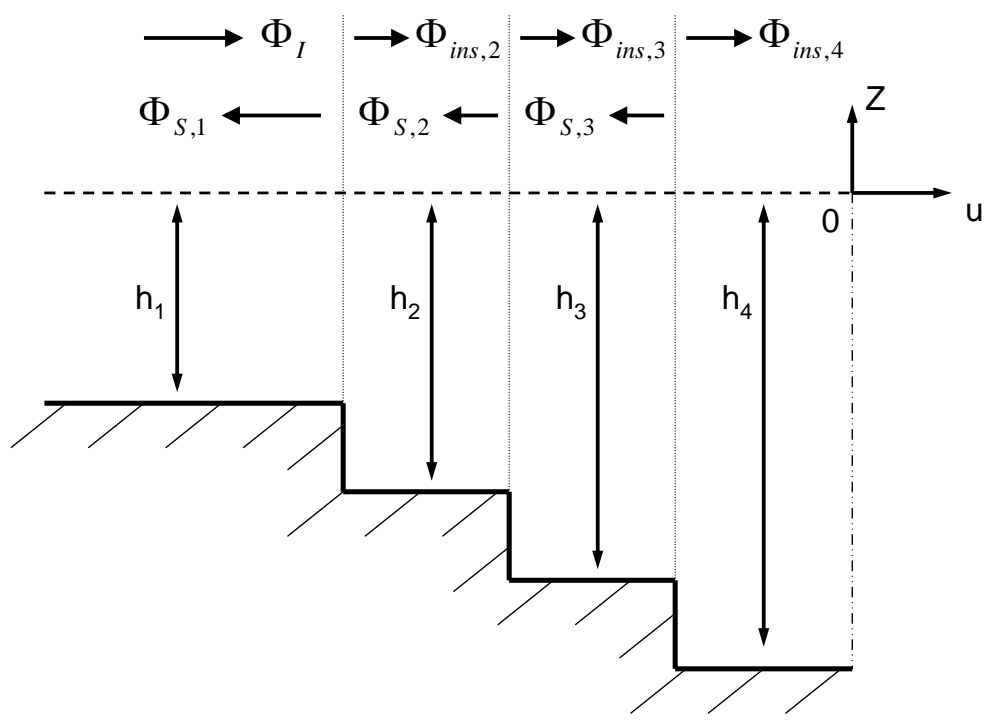

Fig. (2). Definition sketch for boundaries of gradual depth transitions.

domains. The study highlights similarities between Mathieu function behavior and Bessel function solutions in cylindrical domains.

\section{FORMULATION AND THEORY}

The model developed herein investigates the threedimensional motion of monochromatic, small-amplitude water waves in an inviscid and irrotational fluid of arbitrary depth. The waves propagate in an infinite otherwise, uniform depth domain containing a three-dimensional elliptic anomaly (pit or shoal) of finite extent. The elliptic analytic step method is an extension of Bender and Dean [3] — valid for axisymmetric anomalies - that determines the wave transformation in arbitrary water depth for domains in elliptic coordinates with gradual transitions in depth that are approximated by a series of steps of uniform depth. This additional capability to analytically investigate an elliptic form with gradual transitions in depth provides many new, and more practical, possibilities for study compared to the 3-D axisymmetric model domains.
Fig. (1) presents a definition sketch for the case of an elliptic pit with an abrupt depth transition. The parameters $a$ and $b$ define the semi-major and semi-minor axes of the ellipse. The elliptic coefficient, $\mathrm{h}_{\mathrm{c}}$, equals $\left(a^{2}-b^{2}\right)^{0.5}$ and allows transformation from Cartesian $(\mathrm{x}, \mathrm{y})$ to elliptic coordinates $(\mathrm{u}, \mathrm{v})$. The method divides the domain into regions with the bathymetric anomaly and its projection comprising Regions $2 \rightarrow \mathrm{N}_{\mathrm{s}}+1$ where Ns is the number of steps approximating the depth transition slope and the rest of the domain, of depth $\mathrm{h}_{1}$, in Region 1 . For the case of an abrupt transition in depth, the bathymetric anomaly occurs in Region 2 of uniform depth $\mathrm{h}_{2}$, where abrupt is defined as one step either down or up. For the case of a gradual depth transition, steps divide the bathymetric anomaly into subregions with the depth in each subregion equal to $h_{j}$ for each step $j=2 \rightarrow N_{s}+1$.

The step method extends the previous works for abrupt depth transitions by allowing a domain of arbitrary depth that contains a depth anomaly with gradual transitions (sloped sidewalls) between regions. Instead of having a "step 


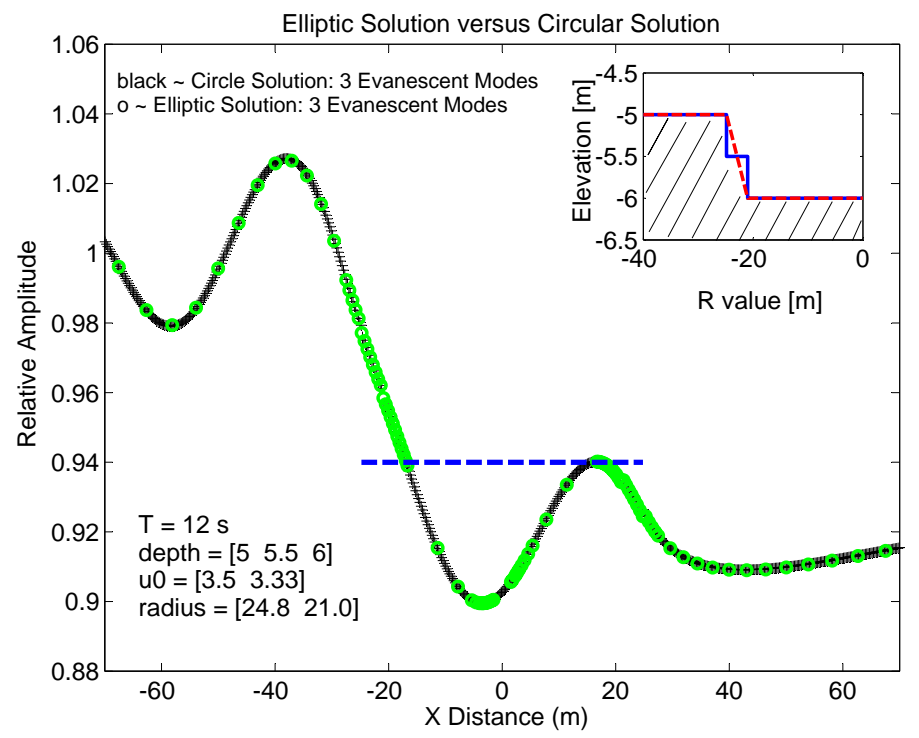

Fig. (3). Comparison of axisymmetric method (Bender and Dean [3]) to elliptic method for case of circular pit with two steps and three evanescent modes; pit geometry shown in inset diagram.

down" from the upwave direction for a pit or a "step up" for the case of a shoal, in the step method a series of steps either up or down, of uniform depth, are connected by a uniform depth region for $\mathrm{u}<\mathrm{u}_{\mathrm{Ns}}$. A sketch of a domain with a stepped pit is shown in Fig. (2) indicating the location and definition of the velocity potentials and boundaries for a pit with $\mathrm{N}_{\mathrm{s}}=$ 3.

Each region has a specified depth and each interface between regions has a specified $u$ location where the matching conditions must be applied. At each boundary the matching conditions depend on whether the boundary is a "step up" or a "step down." Specifying the incident wave allows the formation of a set of equations with $2 \mathrm{~N}_{\mathrm{s}} \mathrm{N}_{\mathrm{e}}(\mathrm{M}+1)$ unknown coefficients where $\mathrm{N}_{\mathrm{e}}$ is the number of non-propagating modes and $\mathrm{M}$ is the number of Mathieu function modes in the solution.

The solution starts with the definition of an incident velocity potential in the form of a plane progressive wave valid in elliptic coordinates:

$$
\begin{aligned}
& \phi_{I}(u, v, z, t) \\
& =M_{I}\left[\begin{array}{c}
\sum_{m=0}^{\infty} \gamma_{m} c e_{m}\left(v, q_{1}\right) c e_{m}\left(\theta, q_{1}\right) J e_{m}\left(u, q_{1}\right)+ \\
\sum_{m=1}^{\infty} \gamma_{m} s e_{m}\left(v, q_{1}\right) s e_{m}\left(\theta, q_{1}\right) J o_{m}\left(u, q_{1}\right)
\end{array}\right] \frac{\cosh \left[k_{1}\left(h_{1}+z\right)\right]}{\cosh \left(k_{1} h_{1}\right)} e^{-i \omega t}(1)
\end{aligned}
$$

where $\gamma_{\mathrm{m}}=1$ for $\mathrm{m}=0$ and $2 \mathrm{i}^{\mathrm{m}}$ otherwise, $\mathrm{M}_{\mathrm{I}}=\frac{-i g H}{2 \omega}$, $\mathrm{u}$ equals the radial distance from the center of the bathymetric anomaly to the point in the fluid domain, $v$ equals the angle to the point measured counter-clockwise from the origin as shown in Fig. (1), $\mathrm{H}$ equals the incident wave height with angle of approach $\theta, \omega$ equals the angular frequency, and $g$ equals the acceleration due to gravity.

The terms $\mathrm{ce}_{\mathrm{m}}$ and $\mathrm{se}_{\mathrm{m}}$ represent even and odd angular (regular) Mathieu functions of the first kind. The $\mathrm{Je}_{\mathrm{m}}$ and $\mathrm{Jo}_{\mathrm{m}}$ terms represent even and odd radial (modified) Mathieu functions of the first kind. Appendix A provides details on the calculation of the angular and radial Mathieu functions.
The scattered velocity potential consists of reflected modes that persist with substantial distance from the pit and evanescent (non-propagating) modes, which decay rapidly with distance from the pit:

$$
\begin{aligned}
& \phi_{S}(u, v, z, t)= \\
& {\left[\begin{array}{c}
\sum_{m=0}^{\infty} A c_{m} c e_{m}\left(v, q_{j}\right) c e_{m}\left(\theta, q_{j}\right) M e_{m}^{1}\left(u, q_{j}\right)+ \\
\sum_{m=1}^{\infty} A s_{m} s e_{m}\left(v, q_{j}\right) s e_{m}\left(\theta, q_{j}\right) M o_{m}^{1}\left(u, q_{j}\right)
\end{array}\right] \frac{\cosh \left[k_{1}\left(h_{1}+z\right)\right]}{\cosh \left(k_{1} h_{1}\right)} e^{-i \omega t}}
\end{aligned}
$$

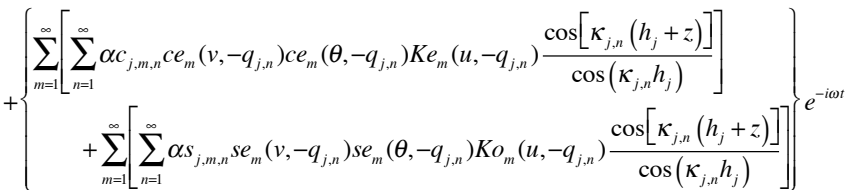

with $\mathrm{Me}_{\mathrm{m}}{ }_{\mathrm{m}}$ indicating the Mathieu-Hankel function of the first kind and $\mathrm{m}^{\text {th }}$ order $-\mathrm{Je}_{\mathrm{m}}+\mathrm{i} \mathrm{Ne} \mathrm{m}_{\mathrm{m}}$ - where the $\mathrm{Ne}_{\mathrm{m}}$ represents the even radial (modified) Mathieu functions of the second kind. The terms $\mathrm{Ke}_{\mathrm{m}}$ and $\mathrm{Ko}_{\mathrm{m}}$ represent even and odd Modified Mathieu equation terms that demonstrate behavior similar to the Modified Bessel equation term $\mathrm{K}_{\mathrm{m}}$. Inside the pit, the velocity potential is given by the form

$$
\begin{aligned}
& \phi_{T}(u, v, z, t)= \\
& {\left[\begin{array}{c}
\sum_{m=0}^{\infty} B c_{m} c e_{m}\left(v, q_{j}\right) c e_{m}\left(\theta, q_{j}\right) J e_{m}\left(u, q_{j}\right)+ \\
\sum_{m=1}^{\infty} B s_{m} s e_{m}\left(v, q_{j}\right) s e_{m}\left(\theta, q_{j}\right) J o_{m}\left(u, q_{j}\right)
\end{array}\right] \frac{\cosh \left[k_{1}\left(h_{1}+z\right)\right]}{\cosh \left(k_{1} h_{1}\right)} e^{-i \omega t}(3)} \\
& +\left\{\begin{array}{c}
\sum_{m=1}^{\infty}\left[\sum_{n=1}^{\infty} \beta c_{j, m, n} c e_{m}\left(v,-q_{j, n}\right) c e_{m}\left(\theta,-q_{j, n}\right) I e_{m}\left(u,-q_{j, n} \frac{\cos \left[\kappa_{j, n}\left(h_{j}+z\right)\right]}{\cos \left(\kappa_{j, n} h_{j}\right)}\right]\right. \\
+\sum_{m=1}^{\infty}\left[\sum_{n=1}^{\infty} \beta s_{j, m, n} s e_{m}\left(v,-q_{j, n}\right) s e_{m}\left(\theta,-q_{j, n}\right) I o_{m}\left(u,-q_{j, n}\right)\right. \\
\cos \left[\kappa_{j, n}\left(h_{j}+z\right)\right] \\
\cos \left(\kappa_{j, n} h_{j}\right)
\end{array}\right]
\end{aligned}
$$

The terms $\mathrm{Ie}_{\mathrm{m}}$ and $\mathrm{Io}_{\mathrm{m}}$ represent even and odd Modified Mathieu equation terms that demonstrate behavior similar to the Modified Bessel equation term $\mathrm{I}_{\mathrm{m}}$. In Eqs. 2 and 3, $A c_{\mathrm{m}}$, 
$\mathrm{As}_{\mathrm{m}}, \mathrm{Bc}_{\mathrm{m}}$, and $\mathrm{Bs}_{\mathrm{m}}$, are unknown amplitude coefficients for the even and odd propagating waves and $\alpha \mathrm{c}_{\mathrm{m}}, \alpha \mathrm{s}_{\mathrm{m}}, \beta \mathrm{c}_{\mathrm{m}}$, and $\beta s_{m}$, are unknown amplitude coefficients for the even and odd non-propagating (evanescent) waves that one must determine for each Mathieu function mode included in the solution.

Table 1. Convergence Test for Elliptic pit with Three Steps Showing Dependence on the Number of Mathieu Function Modes and the Number of Evanescent Modes taken in Solution

\begin{tabular}{|c|c|c|c|c|}
\hline \multicolumn{5}{|c|}{$\begin{array}{c}\text { Elliptic Pit with Three Steps }\left(\mathrm{a} \quad 1=15.8 \mathrm{~m}, \mathrm{~b}_{1}=7.3 \mathrm{~m}\right) \\
\text { Depth }=2,2.3,2.6,3 \mathrm{~m} ; \mathrm{T}=12 \mathrm{~s}, \mathrm{~h} \quad{ }_{\mathrm{c}}=14\end{array}$} \\
\hline $\begin{array}{c}\text { \# of } \\
\text { Mathieu }\end{array}$ & \multicolumn{4}{|c|}{ \# of Evanescent Modes } \\
\hline & 0 & 1 & 2 & 3 \\
\hline 4 & 0.8326 & 0.8326 & 0.8326 & 0.8326 \\
\hline 9 & 1.2875 & 1.2878 & 1.2880 & 1.2881 \\
\hline 14 & 1.0054 & 1.0057 & 1.0060 & 1.0061 \\
\hline 18 & 0.9487 & 0.9490 & 0.9492 & 0.9493 \\
\hline 22 & 0.9465 & 0.9468 & 0.9470 & 0.9471 \\
\hline 26 & 0.9465 & 0.9468 & 0.9470 & 0.9471 \\
\hline 30 & 0.9465 & 0.9468 & 0.9470 & 0.9471 \\
\hline 34 & 0.9465 & 0.9468 & 0.9470 & 0.9471 \\
\hline 38 & 0.9470 & 0.9473 & 0.9475 & 0.9476 \\
\hline 42 & 0.9849 & 0.9853 & 0.9855 & 0.9856 \\
\hline 46 & 7.1967 & 7.1972 & 7.1974 & 7.1976 \\
\hline 50 & 297.9236 & 297.9241 & 297.9243 & 297.9244 \\
\hline
\end{tabular}

Matching conditions for the velocity potential and horizontal velocity with depth at the interfaces $\left(\mathrm{u}=\mathrm{U}_{\mathrm{j}}\right)$ between regions allow solution of the equations via truncated series. Applying the matching conditions results in a truncated set of independent integral equations each of which is multiplied by the appropriate eigenfunction; $\cosh \left[\mathrm{k}_{\mathrm{j}}\left(\mathrm{h}_{\mathrm{j}}+\mathrm{z}\right)\right]$ or $\cos \left[\kappa_{\mathrm{j}, \mathrm{n}}\left(\mathrm{h}_{\mathrm{j}}+\mathrm{z}\right)\right]$. The proper eigenfunction to use depends on whether the boundary results in a "step down" or a "step up" as $\mathrm{u}$ approaches zero; thereby making the form of the solution for a pit different than that of a shoal.

At each boundary $\left(\mathrm{U}_{\mathrm{j}}\right)$ the appropriate evanescent mode contributions from the adjacent boundaries $\left(\mathrm{U}_{\mathrm{j}-1}, \mathrm{U}_{\mathrm{j}+1}\right)$ are taken into account. The resulting set of simultaneous equations is solved as a linear matrix equation with the values of $\mathrm{M}, \mathrm{N}_{\mathrm{e}}$ and $\mathrm{N}_{\mathrm{s}}$ sufficiently large - approximately 40,3, and 3 (linear slope) for the cases presented - to ensure convergence of the solution.

The numerical solution of the Mathieu and Modified Mathieu functions applies numerical programs and theory from Zhang and Jin [14]. Specifically the method employs expansion coefficients calculated with computer code from Zhang and Jin [14] and applies these expansion coefficients to develop values of the Mathieu and Modified Mathieu functions. The Appendix contains further details of the equations applied.

\section{RESULTS}

Solution of the three-dimensional models establishes the complex velocity potential anywhere in the fluid domain. The complex velocity potential allows calculation of the wave height and direction accounting for the wave transformation processes of refraction, diffraction, and reflection that act on a planar wave when encountering a depth transition. The modeling effort focused on the effect of the variation in the longshore and cross-shore extent of the elliptic form on the wave field modification. The study includes depth anomalies with gradual transitions in depth to more accurately represent forms found in nature.

\section{COMPARISON TO AXISYMMETRIC SOLUTION}

Comparing the model developed herein for an elliptic form with equal semi-major and semi-minor axes (circle) to the model of Bender and Dean [3] — valid for axisymmetric depth anomalies - provides verification of the elliptic model. Fig. (3) presents results for both the elliptic model and the model from Bender and Dean [3] shown as relative amplitude for a pit of circular form with the pit extent shown by the dashed line. For this comparison the pit has two steps with inner and outer radii of 21.0 and $24.8 \mathrm{~m}$. The inset figure indicates a cross-section of the pit geometry through the centerline. The main figure indicates almost identical solutions upwave, downwave and inside of the pit. Other comparisons with different circular geometries indicate similar agreement in the relative amplitude values.

\section{CONVERGENCE OF ELLIPTIC STEP METHOD}

Application of the Mathieu function solutions developed reveals convergence to a stable solution for up to approximately 30 Mathieu function modes. Table 1 presents results of tests completed for the case of an elliptic pit with $\mathrm{a}_{1}=$ $15.8 \mathrm{~m}, \mathrm{~b}_{1}=7.3 \mathrm{~m}$ and three steps from $2 \mathrm{~m}$ depth to $3 \mathrm{~m}$ depth. For $0,1,2$, and 3 evanescent modes, the table presents relative amplitude values at a location $100 \mathrm{~m}$ downwave of the ellipse center for an incident wave period of $12 \mathrm{~s}$. For this configuration and wave conditions, the solution shows convergence as the number of evanescent modes increases with little improvement from 2 to 3 modes. The solution stabilizes for cases with more than 22 Mathieu function modes. However, more than 34 Mathieu function modes cause the solution to deviate and for greater than 46 modes the solution begins to diverge significantly. Convergence problems for higher orders of Mathieu functions are not an uncommon problem. The methods developed for the applications presented in this paper allow solution prior to convergence problems degrading the solution.

\section{ELLIPTIC PIT}

The following figures demonstrate the wave transformation caused by elliptic pits with various side slopes as plots of the relative wave amplitude. The depth anomaly consists of five ellipses of uniform depth with the largest having a semi-major axis (a) length of $33 \mathrm{~m}$ and a semi-minor axis (b) length of $16 \mathrm{~m}$. Fig. (4) presents a planform view of the elliptic anomaly. The slopes of the depth transitions equal 1:3 and 1:10 for the semi-major and semi-minor axes. The domain has a uniform depth of $2 \mathrm{~m}$ outside the depth anomaly and depths of 2.2, 2.4, 2.6, 2.8 and $3 \mathrm{~m}$ for each elliptical step. Fig. (5) presents transects parallel to the $\mathrm{x}$ - and $\mathrm{y}$-axis through the centerline of the elliptic form.

Fig. (6) presents a contour plot of the wave amplitude field normalized by the incident wave amplitude for an ellip- 


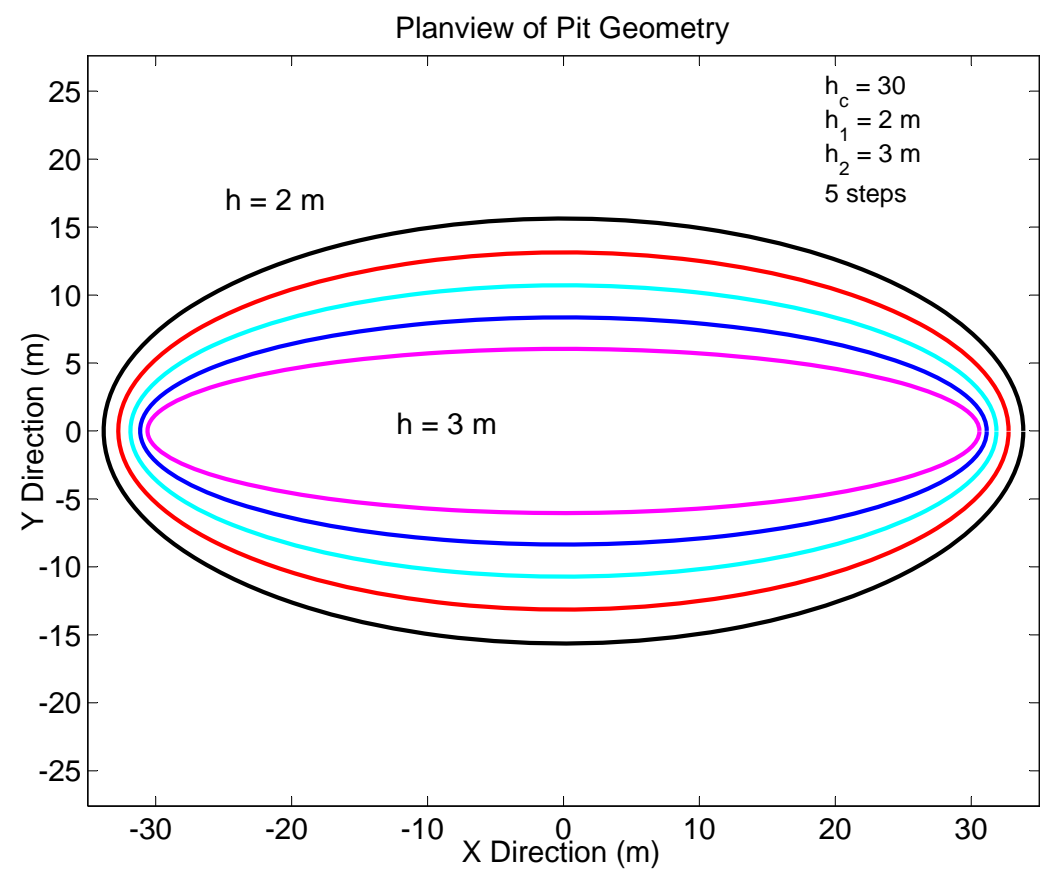

Fig. (4). Planform view of elliptic pit applied in wave transformation modeling; specifics of pit geometry listed in figure.
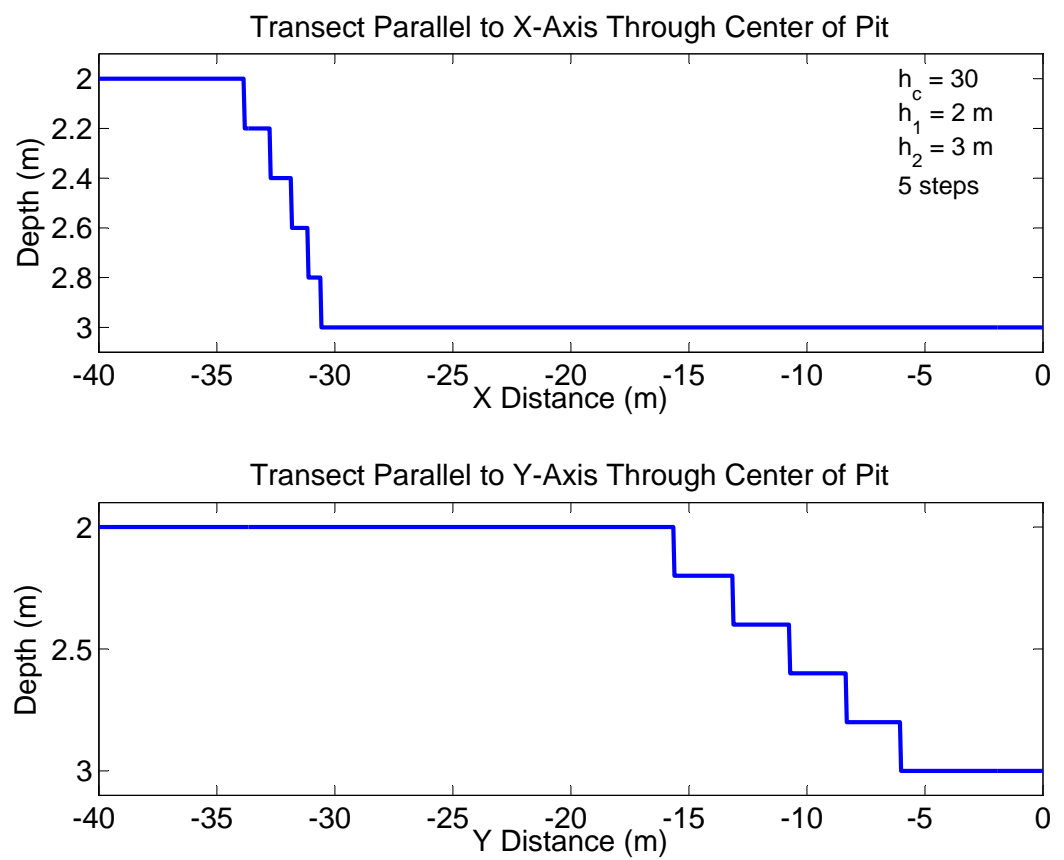

Fig. (5). Cross-sectional view taken along semi-major and semi-minor axes of elliptic pit (planview in Fig. 4) applied in wave transformation modeling; specifics of pit geometry listed in figure.

tic pit with sloped sidewalls as shown in Figs. (4 and 5). In Fig. (6) the incident waves propagate from left to right, parallel to the $\mathrm{x}$-axis (major) labeled in the figure. This figure clearly shows the divergence of the wave field caused by the pit with a large area of wave sheltering evident directly shoreward of the pit. Two bands of relative wave amplitude greater than one, from the converging wave field at these locations, flank this area. The relative amplitude values show symmetry about $\mathrm{Y}=0$.

Employing an oblique incident wave angle in a domain with an elliptic depth anomaly creates asymmetry in the re- sulting wave field. The asymmetry develops from the interaction of the even and odd Mathieu and Modified Mathieu function solutions. Fig. (7) presents a contour plot of the relative wave amplitude for an incident wave angle of 45 degrees with the same depth anomaly as in Fig. (6). Fig. (7) contains similar features as those of the previous figure with an area of wave sheltering downwave of the depth anomaly flanked by two areas of increased relative amplitude.

Applying an incident wave traveling in the direction of the semi-minor axis demonstrates the influence of the elliptic shape on the wave field. Fig. (8) presents a contour plot of 


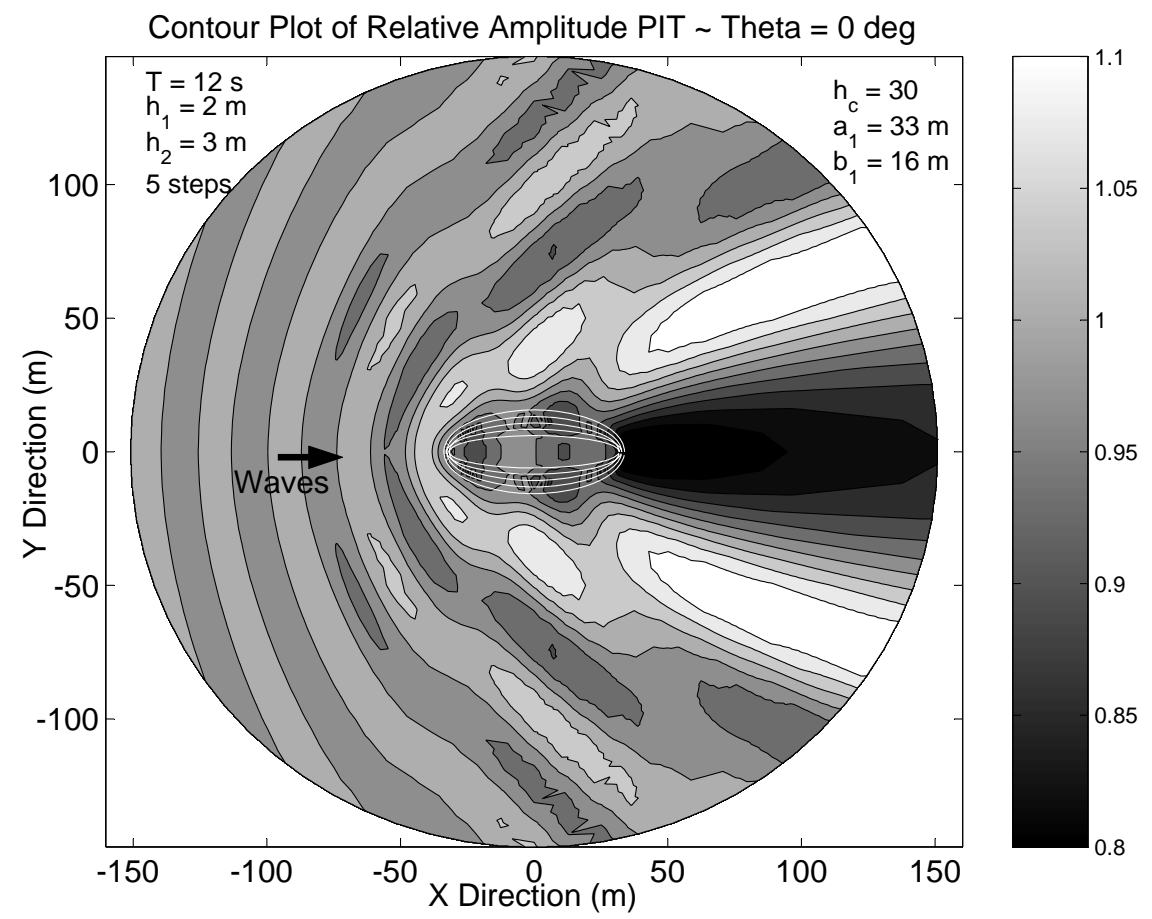

Fig. (6). Contour plot of relative wave amplitude for elliptic pit with five depth transitions and incident wave angle equal to 0 degrees; labels on figure provide model parameters.

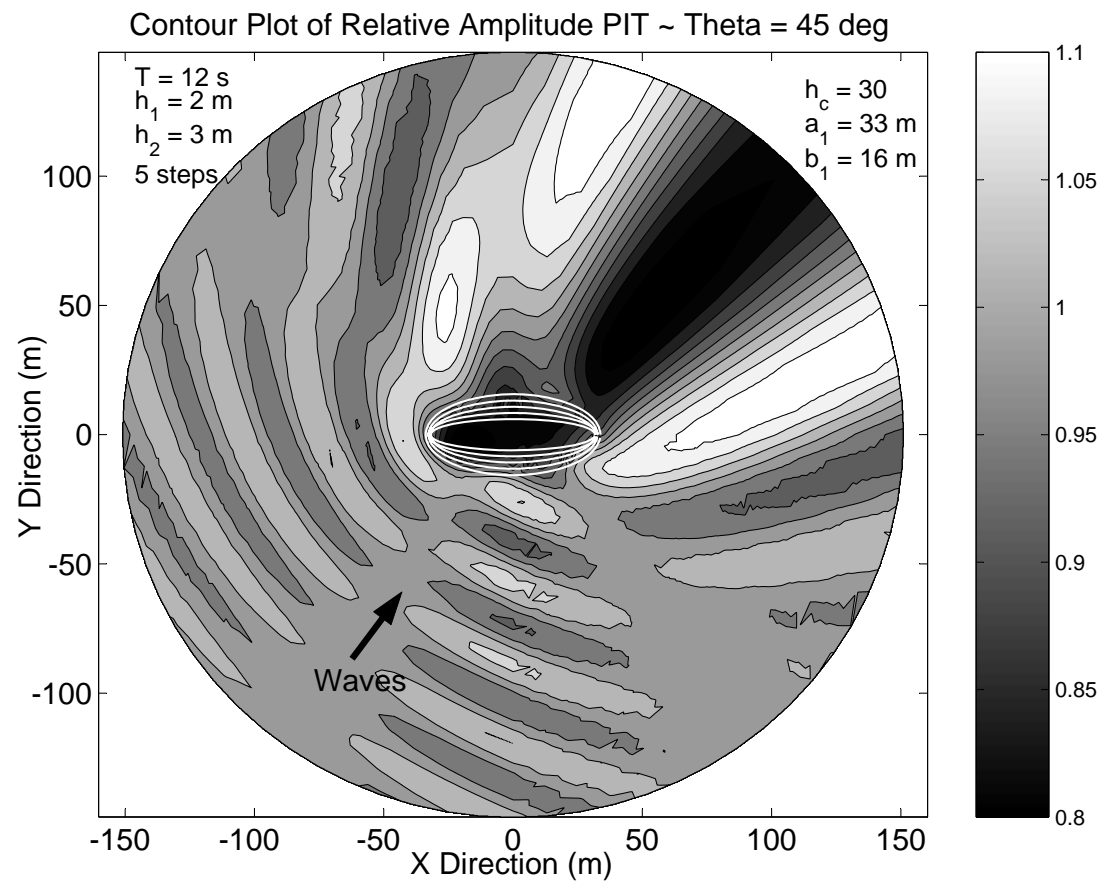

Fig. (7). Contour plot of relative wave amplitude for elliptic pit with five depth transitions and incident wave angle equal to 45 degrees; labels on figure provide model parameters.

the relative amplitude for an incident angle of 90 degrees with the same depth anomaly as the two previous figures. A symmetric wave pattern develops with increased reflection and downwave sheltering as compared to the conditions of Fig. (6).

Fig. (9) presents the relative amplitude along transects taken in the direction of wave propagation through the center of the elliptical forms for incident angles of 0,45 , and 90 degrees. The figure highlights the influence of the elliptic shape on the wave transformation processes resulting from the interaction of the wave field with the depth anomaly. To focus attention on the relative amplitudes outside of the elliptic form, Fig. (9) and following plots with results along a transect present results outside of the interior of the bathymetric anomaly (pit or shoal). To facilitate comparison 


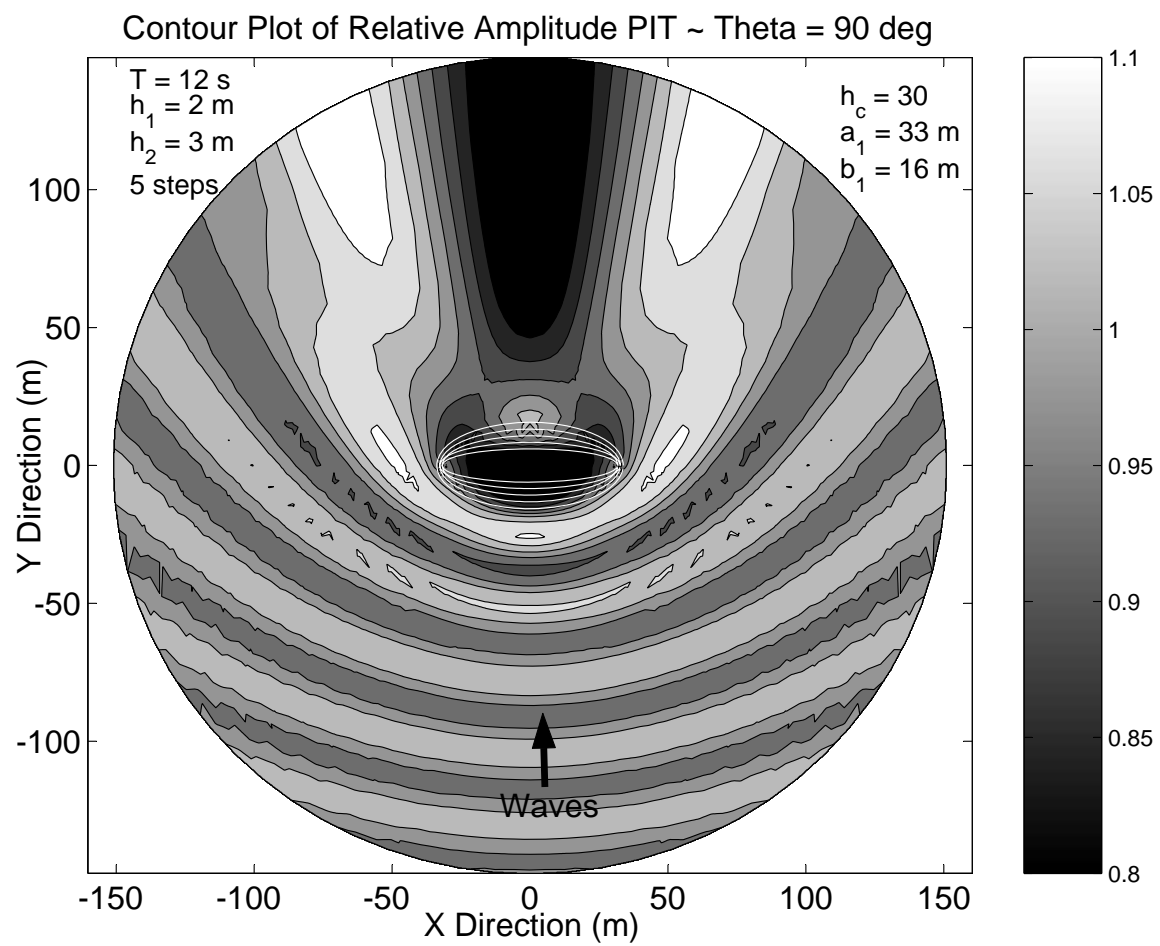

Fig. (8). Contour plot of relative wave amplitude for elliptic pit with five depth transitions and incident wave angle equal to 90 degrees; labels on figure provide model parameters.

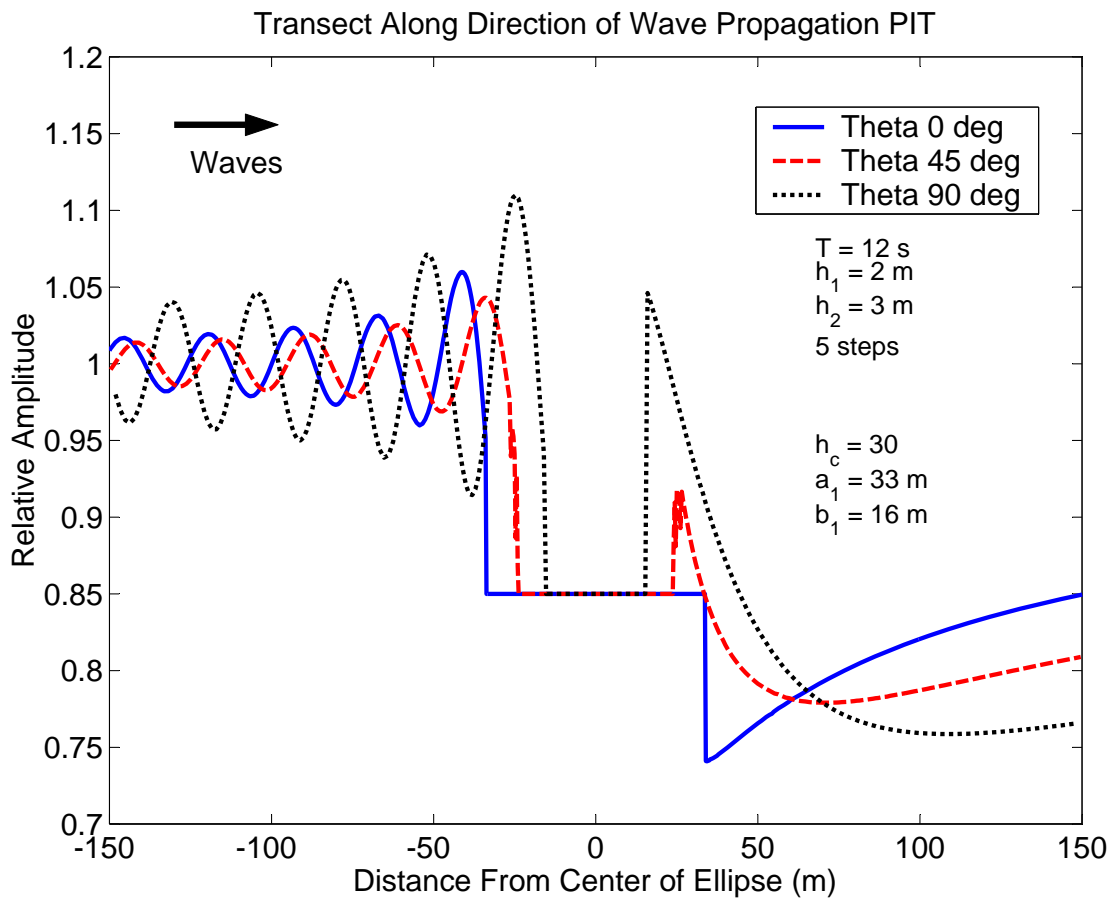

Fig. (9). Relative wave amplitude along transects in the direction of wave propagation $(0,45$, and 90 deg) through the center of the elliptic pit; labels on figure provide model parameters.

between the three cases, the plots along the $\mathrm{x}$-axis in Fig. (10) are shifted relative to the semi-major axis of the largest ellipse for each case, $\mathrm{a}_{1}$. The transect plots only present relative amplitude values.

With an incident angle of 90 degrees the wave field encounters a depth anomaly that is much wider relative to the wave direction resulting in larger reflection and sheltering than for the case of a 0 deg incident wave, which faces a more streamlined depth anomaly. A unique behavior of the wave incident from 90 degrees is a relative amplitude greater than one on the downwave edge of the pit. For this case the relative amplitude is shown to decrease dramatically as downwave distance from the pit increases. 


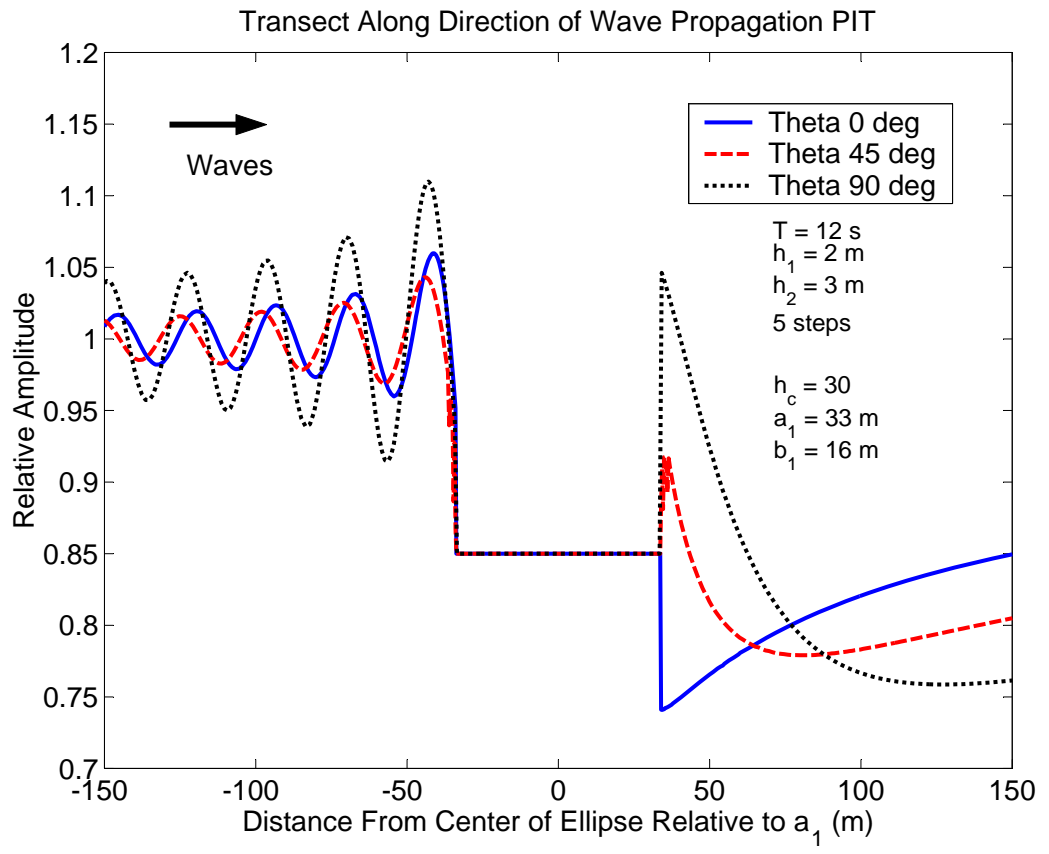

Fig. (10). Relative wave amplitude along transects in the direction of wave propagation $(0,45$, and 90 deg) through the center of the elliptic pit shifted to $a_{1}$; labels on figure provide model parameters.

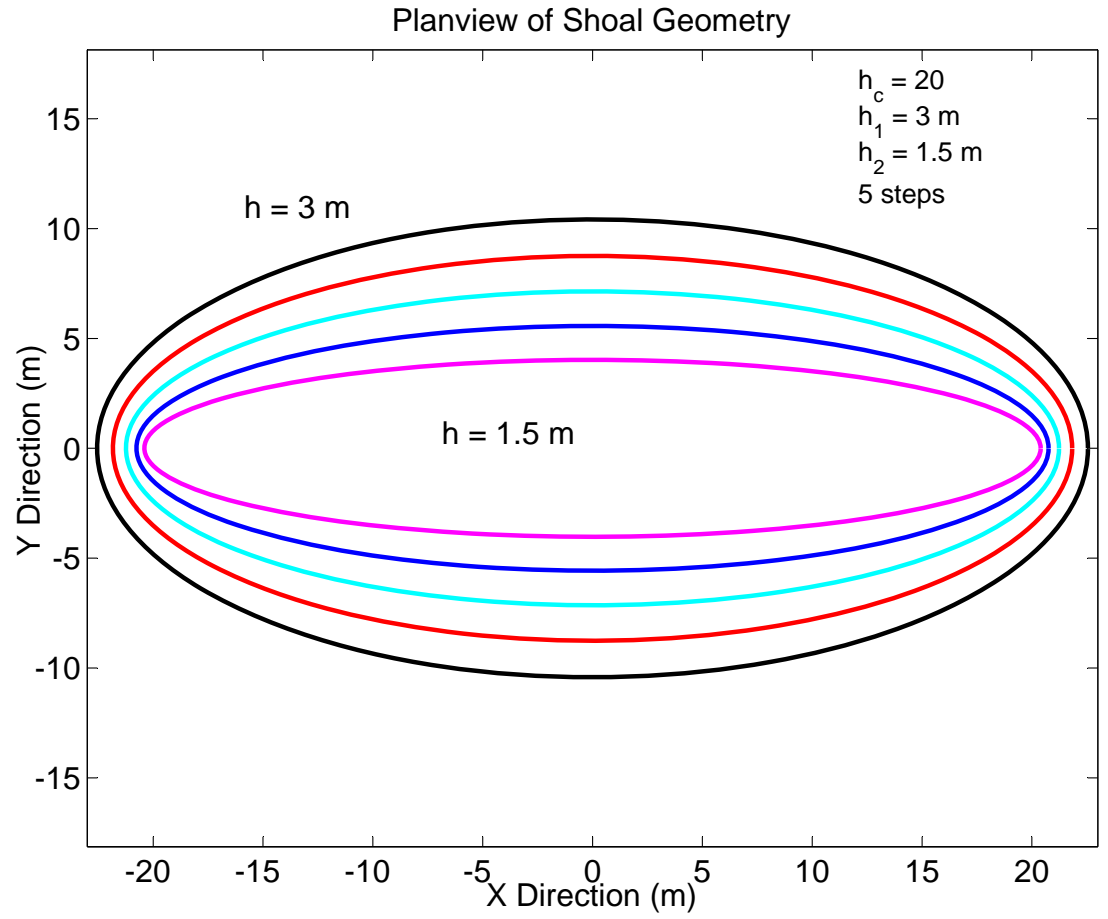

Fig. (11). Planform view of elliptic shoal applied in wave transformation modeling; specifics of shoal geometry listed in figure.

\section{ELLIPTIC SHOAL}

The method developed also allows investigation of bathymetric anomalies in the form of elliptic shoals with gradual transitions in depth. Figs. (11 and 12) present planform and cross-sectional views of the shoal geometry applied in the following figures. The depth anomaly consists of five ellipses of uniform depth with the largest having a semimajor axis (a) length of $22.6 \mathrm{~m}$ and a semi-minor axis (b) length of $10.4 \mathrm{~m}$. The transitions in depth are $1: 3$ for the semi-major axis and 1:6 for the semi-minor axis. The domain has a uniform depth of $3 \mathrm{~m}$ outside the depth anomaly and depths of 2.7, 2.4, 2.1, 1.8 and $1.5 \mathrm{~m}$ for each elliptical step that comprise the shoal.

Fig. (13) presents a contour plot of the relative amplitude for an elliptic shoal with sloped sidewalls. In this figure the incident waves propagate with an angle of 45 degrees. The convergence of the wave field caused by the shoal is shown clearly in Fig. (13) with a large area of wave focusing evi- 
Transect Parallel to X-Axis Through Center of Shoal

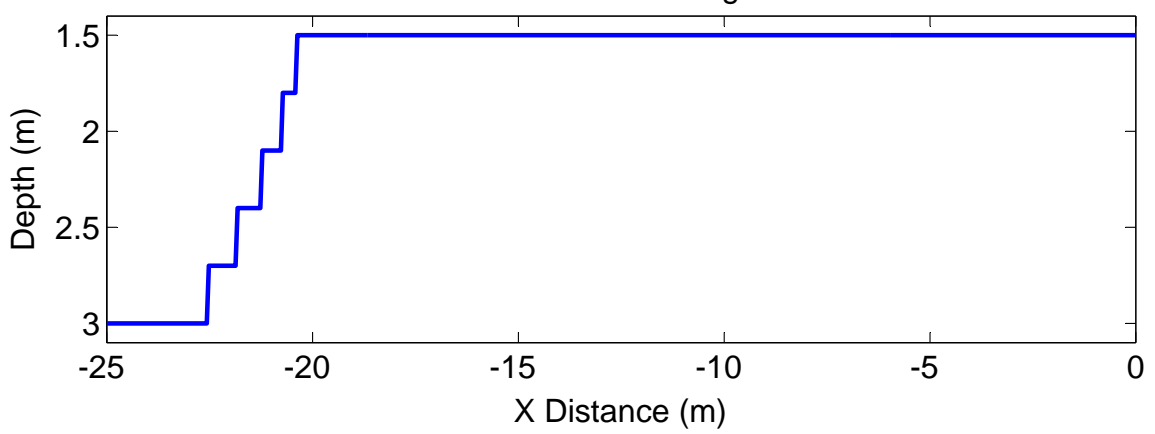

Transect Parallel to Y-Axis Through Center of Shoal

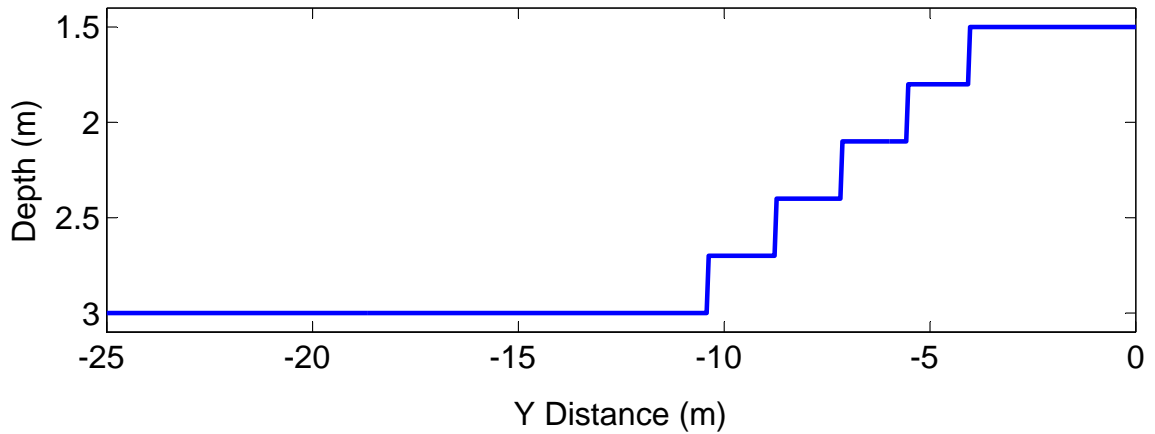

Fig. (12). Cross-sectional view taken along semi-major and semi-minor axes of elliptic shoal (shown in Fig. 11) applied in wave transformation modeling; specifics of shoal geometry listed in figure.

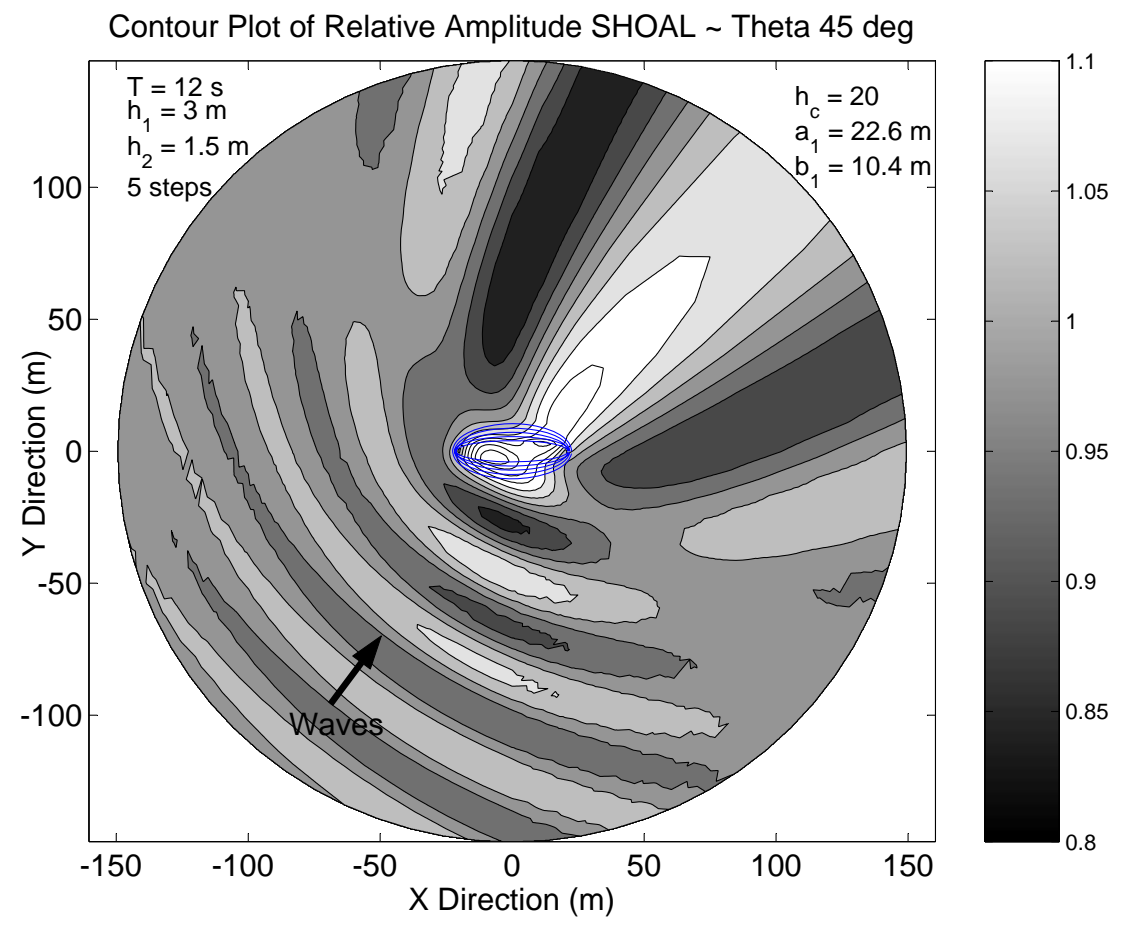

Fig. (13). Contour plot of relative wave amplitude for elliptic shoal with five depth transitions and incident wave angle equal to 45 degrees; labels on figure provide model parameters.

dent downwave of the shoal. Two bands of relative wave amplitude less than one, caused by the diverging wave field, flank this area.

Fig. (14) presents a comparison of the relative amplitude along transects in the direction of wave propagation and through the center of the elliptical forms for three incident wave angles - 0, 45, and 90 degrees. To facilitate comparison between the three cases the distance along the $\mathrm{x}$-axis is shifted relative to the semi-major axis of the largest ellipse for each case, $a_{1}$. This figure indicates a significant difference in the reflection for an incident wave angle of 90 degrees compared to 0 degrees. With the shape of the elliptic 


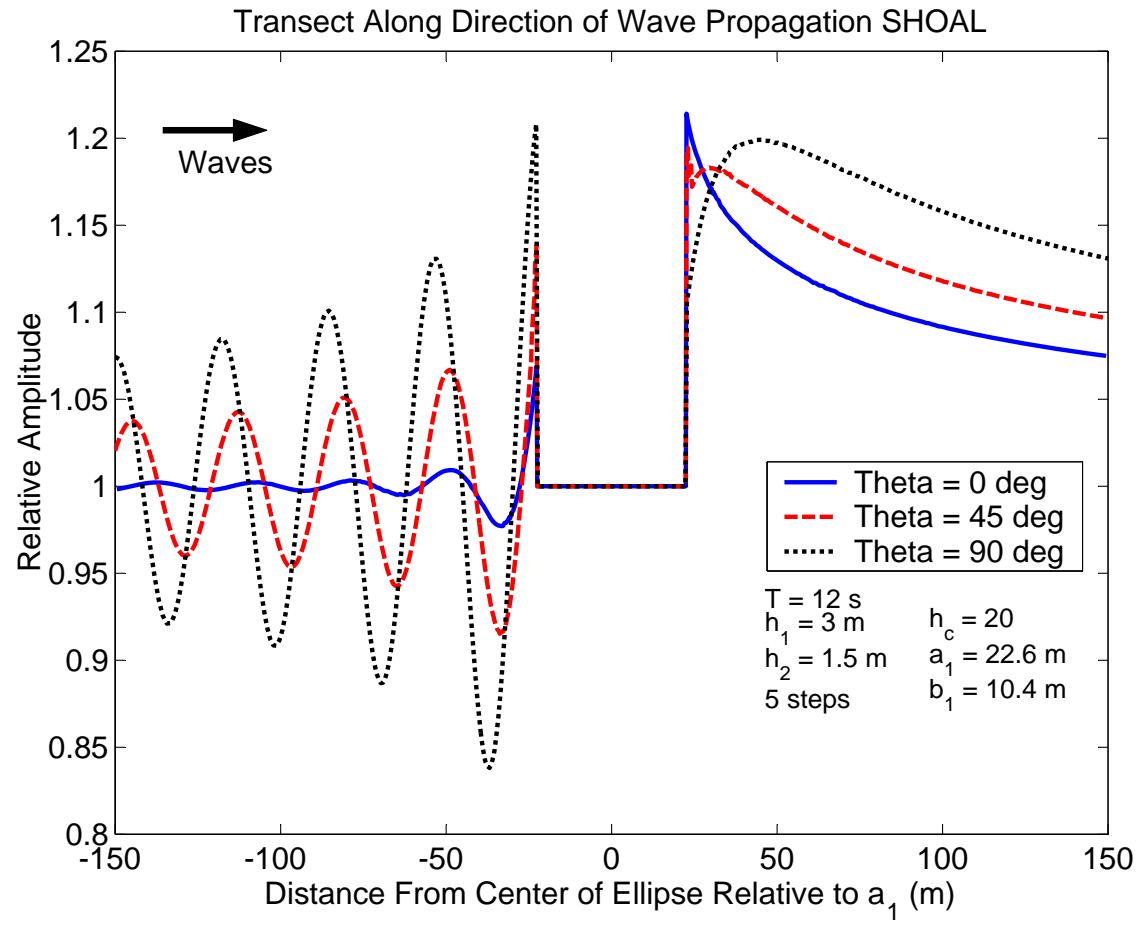

Fig. (14). Relative wave amplitude along transects in the direction of wave propagation $(0,45$, and 90 deg) through the center of the elliptic shoal shifted to $\mathrm{a}_{1}$; labels on figure provide model parameters.

shoal, waves propagating from 90 degrees encounter an obstruction $1.5 \mathrm{~m}$ below the water surface and $40 \mathrm{~m}$ wide. In comparison, waves propagating from 0 degrees encounter an obstruction only $8 \mathrm{~m}$ wide at the same depth.

\section{DISCUSSION AND CONCLUSIONS}

The elliptic analytic (semi-numerical) step method determines the wave field in the presence of an elliptic bathymetric anomaly with gradual transitions in depth and incorporating the wave transformation processes of refraction, diffraction, and reflection. Varying the incident wave angle allows one to view the influence of the elliptic form on wave transformation. For the cases presented, depth anomalies with their major axes parallel to wave crests result in larger reflection and sheltering than for depth anomalies with their major axes perpendicular to the wave crests.

Future work will compare the elliptic analytic step method to more complex, numerical models that allow arbitrary bathymetry. Additionally, coupling the transformed wave fields landward of the depth anomalies to a simple shoreline change model will allow investigation of shoreline impacts caused by elliptic depth anomalies.

\section{APPENDIX}

Zhang and Jin [14] present the Mathieu and Modified Mathieu function equations employed to develop the results applied in the model. Please consult Zhang and Jin [14] for the full set of equations; a brief list of the even functions of order $2 \mathrm{n}$ follows:

$$
c e_{2 n}=\sum_{j=1}^{\infty} A e_{j}(q) \cos (2[j-1] v)
$$

$$
\begin{aligned}
& s e_{2 n+2}=\sum_{j=1}^{\infty} B e_{j}(q) \sin ([2(j-1)+2] v) \\
& J e_{2 n}=\frac{1}{A_{0}} \sum_{j=1}^{\infty}(-1)^{j-1+n} A e_{j} J_{j-1}\left(v_{1}\right) J_{j-1}\left(v_{2}\right) \\
& J o_{2 n+2}=\frac{-1}{B_{2}} \sum_{j=1}^{\infty}(-1)^{j-1+n} B e_{j}\left(\left[J_{j-1}\left(v_{1}\right) J_{j-1+2}\left(v_{2}\right)\right]-\left[J_{j-1+2}\left(v_{1}\right) J_{j-1}\left(v_{2}\right)\right]\right) \\
& N e_{2 n}=\frac{1}{A_{0}} \sum_{j=1}^{\infty}(-1)^{j-1+n} A e_{j} J_{j-1}\left(v_{1}\right) Y_{j-1}\left(v_{2}\right) \\
& N o_{2 n+2}=\frac{-1}{B_{2}} \sum_{j=1}^{\infty}(-1)^{j-1+n} B e_{j}\left(\left[J_{j-1}\left(v_{1}\right) Y_{j-1+2}\left(v_{2}\right)\right]-\left[J_{j-1+2}\left(v_{1}\right) Y_{j-1}\left(v_{2}\right)\right]\right) \\
& K e_{2 n}=\frac{1}{A_{0}} \sum_{j=1}^{\infty} A e_{j} I_{j-1}\left(v_{1}\right) K_{j-1}\left(v_{2}\right) \\
& I o_{2 n+2}=\frac{1}{A_{0}} \sum_{j=1}^{\infty}(-1)^{j-1+n} A e_{j} I_{j-1}\left(v_{1}\right) I_{j-1}\left(v_{2}\right) \\
& =\frac{1}{B_{2}} \sum_{j=1}^{\infty} B e_{j}\left(\left[I_{j-1}\left(v_{1}\right) K_{j-1+2}\left(v_{2}\right)\right]-\left[I_{j-1+2}\left(v_{1}\right) K_{j-1}\left(v_{2}\right)\right]\right) \\
& (\mathrm{A} .6) \\
& B e_{j}\left(\left[I_{j-1}\left(v_{1}\right) I_{j-1+2}\left(v_{2}\right)\right]-\left[I_{j-1+2}\left(v_{1}\right) I_{j-1}\left(v_{2}\right)\right]\right)
\end{aligned}
$$


In Equations A.1 - A.10, $\mathrm{n}$ equals the Mathieu function order developed with summation of $\mathrm{j}$ terms, Ae and Be equal the expansion coefficients; $\mathrm{v}$ equals the angle to the point measured counter-clockwise from the origin as shown in Fig. (1); $v_{1}$ equals $(q)^{0.5} \mathrm{e}^{-\mathrm{u}} ; v_{2}$ equals $(\mathrm{q})^{0.5} \mathrm{e}^{\mathrm{u}} ; \mathrm{q}$ equals $(\mathrm{kh})^{2} / 2$ where $k$ equals the wave number and $h$ equals the elliptic coefficient; $u$ equals the radial distance from the center of the bathymetric anomaly to the point in the fluid domain; $\mathrm{J}$ and $\mathrm{Y}$ equal Bessel functions, and I and $\mathrm{K}$ equal modified Bessel functions.

\section{REFERENCES}

[1] K. Takano, "Effets d'un obstacle paraléllélépipédique sur la propagation de la houle", Houille Blanche, vol. 15, pp. 247, 1960.

[2] C.J. Bender and R.G. Dean, "Potential shoreline changes induced by three-dimensional bathymetric anomalies with gradual transitions in depth", Coastal Eng., vol. 51, no. 11-12, pp. 1143-1163, 2004.

[3] C.J. Bender and R.G. Dean, "Wave transformation by axisymmetric three-dimensional bathymetric anomalies with gradual transitions in depth" Coastal Eng., vol. 52, no. 4, pp. 331-351, 2005.

[4] A.J. Combe and C.W. Soileau, "Behavior of man-made beach and dune: Grand Isle, Louisiana", Proceedings of Coastal Sediments 1987, New Orleans, Louisiana, pp. 1232-1242, 1987.
[5] B.P. Williams, Physical modeling of nearshore response to offshore borrow pits, Masters Thesis, Department of Civil and Coastal Engineering, University of Florida, 2002.

[6] K. Horikawa, T. Sasaki, and H. Sakuramoto, "Mathematical and laboratory models of shoreline change due to dredged holes", $J$. Faculty Eng. Univ. Tokyo, vol. 34, no. 1, pp. 49-57, 1977.

[7] J.M. Motyka and D.H. Willis, "The effect of refraction over dredged holes", Proceedings 14th International Conference on Coastal Engineering, Copenhagen, pp. 615-625, 1974.

[8] J.L Black and C.C. Mei, "Scattering and radiation of water waves", Technical Report No. 121, Water Resources and Hydrodynamics Laboratory, Massachusetts Institute of Technology, 1970.

[9] A.N. Williams, "Diffraction of long waves by a rectangular pit", $J$. Waterway, Port, Coastal, Ocean Eng., vol. 116, pp. 459-469, 1990.

[10] A.N. Williams and J.H. Vasquez, "Wave interaction with a rectangular pit", J. Offshore Mechanics Arctic Eng., vol. 113, pp. 193198, 1991.

[11] W.G. McDougal, A.N. Williams, and K. Furukawa, "Multiple pit breakwaters", J. Waterway, Port, Coastal, Ocean Eng., vol. 122, pp. 27-33, 1996

[12] H.S. Chen and C.C. Mei, "Wave forces on a stationary platform of elliptical shape", J. Ship Res., vol. 17, no. 2, pp. 61-71, 1973.

[13] J.C. Gutiérrez-Vega, R. M. Rodríguez-Dagnino, M. A. MenesesNava, and S. Chávez-Cerda, "Mathieu functions, a visual approach", Am. J. Phys., vol. 71, no. 3, pp. 233-242, 2003.

[14] S. Zhang and J. Jin, "Computation of special functions", John Wiley and Sons: New York, p. 717, 1996.

Received: January 27, 2011

(C) Bender and Dean; Licensee Bentham Open.

This is an open access article licensed under the terms of the Creative Commons Attribution Non-Commercial License (http://creativecommons.org/licenses/by-nc/3.0/) which permits unrestricted, non-commercial use, distribution and reproduction in any medium, provided the work is properly cited. 